\title{
SOBRE A LÓGICA DEÔNTICA NÃO-CLÁSSICA
}

LEILA Z. PUGA

NEWTON C.A. DA COSTA

Universidad Cat6lica de Sao Paulo

y Universidad de Sao Paulo

\section{Introdução}

Mais ou menos até princípios deste século, havia uma única logica (formal, pura ou térica). Mas, no decurso dos últimos oitenta anos, foram criadas outras, de modo que a logica inicialmente considerada passou a ser chamada de logica clássica ou tradicional. Esta se caracteriza por determinados princípios básicos, de natureza sintática e semântica. Quando tais princípios são derrogados ou se amplia o corpo dos conceitos sintáticos e semânticos envolvidos, nascem as chamadas lógicas não-clássicas.

As lógicas não-clássicas dividem-se em duas categorias, a saber: 1) as rivais da lógica clássica; e 2) as complementares da lógica clássica.

Dentre as leis que vigem na logica clássica, há três que sāo importantes, especialmente do ponto de vista histórico: a lei da indentidade (todo objeto é idêntico a si mesmo), a lei da contradição (de duas proposiçōes contraditorítias, isto é, uma das quais é a negação da outra, uma delas é falsa) e a lei do terceiro excluído (de duas proposições contraditórias, uma delas é verdadeira).

As logicas rivais da clássica, mais importantes, caracterizam-se, essencialmente, por derrogarem uma dessas três leis, pelo menos.

Há sistemas lógicos, pois, nos quais a lei da identidade não, é, em geral, válida. Como essa lei também se denomina lei reflexiva da identidade, tais lógicas são denominadas l6gicas não-reflexivas.

Chama-se paracompleta uma lógica que derroga a lei do 
terceiro excluído. Em teorias fundamentadas em tais logicas, pode haver proposições tais que nem elas nem elas nem suas negações sejam verdadeiras. Exemplo de lógica paracompleta é a logica intuicionista, de Brouwer-Heyting.

Infringe-se a lei da contradição na maioria das lógicas chamadas paraconsistentes.

Para melhor conceituarmos os sistemas paraconsistentes, necessitamos de alguns esclarecimentos preliminares. Uma teoria dedutiva $\mathrm{T}$ diz-se inconsistente se entre os seus teoremas há pelo menos dois, um dos quais é a negação do outro; em caso contrário, $T$ denomina-se consistente. A teoria $T$ diz-se trivial (ou supercompleta) quando todas as sentenças formuláveis em sua linguagem forem teoremas dessa teoria; em caso contrário, $T$ chama-se não-trivial.

Um dos traços marcantes da lógica clássica é o de que qualquer teoria dedutiva, nela baseada, que for inconsistente, será, também, trivial e inversamente. Essa lógica não permite que se separem os conceitos de trivialidade e de inconsistência.

Pode-se definir a logica paraconsistente como a lógica que não exclui teorias inconsistentes e não-triviais. Maiores detalhes sobre essas logicas podem ser encontradas em da Costa 1974, em Loparic e da Costa 1984 e em Arruda 1980.

As lógicas complementares da clássica não colocam em xeque os princípios basilares da mesma; de fato, elas ampliam as idéias desta última, introduzindo modalidades e outras noçбes que a tornam mais potente conceitualmente. Como exemplo de lógicas complementares da clássica, lembraremos as lógicas modal (alética), do tempo e epistêmica.

Aqui estamos particularmente interessados na lógica deôntica. Nela nos ocupamos de operadores que podem ser acrescentados à clássica, e que correspondem às noções de obrigatório, permitido, proibido e indiferente. Assim encarada, a lógica deôntica é uma lógica complementar da clássica.

No entanto, podemos, también, desenvolver sistemas de lógica deôntica baseados em lógicas rivais da clássica; tais 
sistemas deônticos são, por conseguinte, rivais da lógica deôntica clássica. Deste modo, podemos estudar sistemas de lógica deôntica para completos e paraconsistentes.

A lógica deôntica, tanto clássica como não-clássica, achase estreitamente ligada à ética e ao direito. Em nossa época, a lógica deôntica foi investigada por E. Mally, em 1926, mas o seu desenvolvimento atual começou com um artigo pioneiro de G. H. von Wright em 1951 (sobre a história da lógica deôntica, pode-se consultar Hilpinen 1971 e Hilpinen 1981).

A finalidade deste artigo, basicamente expositivo, é a de apresentar, de modo sistemático, alguns resultados que obtivemos no âmbito da lógica deôntica. Nossa exposiçáo, embora auto-suficiente, pressupõe da parte do leitor algum conhecimento de lógica deôntica; ela está calcada nos seguintes trabalhos: da Costa e Carnielli 198+, Puga et al. $198+$ e Puga 1985.

\section{Gramática da Lógica Deôntica}

Usualmente, interpretamos as sentenças deônticas, digamos éticas, como prescritivas. Elas prescrevem o que deve ser feito, o que é permitido, etc. Em outras palavras, a lógica deôntica acha-se intimamente relacionada com imperativos e ações.

Não obstante, há uma outra maneira de se interpretar as proposições deônticas: elas descreveriam aquilo que é permitido, obrigatório, etc., em determinado sistema normativo. A lógica deôntica, então, investiga as relações lógicas entre tais enunciados. Assim se obtém um tratamento descritivo da lógica em apreco.

Como normas ou ações não possuem valor de verdade, ao passo que os enunciados descritivos correspondentes se comportam como proposições comuns, o equacionamento descritivo da lógica deôntica encontra-se mais próximo dos sistemas lógicos usuais. Esta é uma das razões que nos induzem a adotar aqui a posição descritiva. Porém, as posiçōes descritiva e prescritiva não se excluem, mas, ao contrário, se 
completam mutualmente. Em trabalhos futuros, tencionamos abordar a lógica deôntica, como é aqui tratada, de um ponto de vista prescritivo, o que nos levará a construir uma lógica da ação e dos imperativos.

Em síntese, neste artigo, a logica deôntica, clássica ou não-clássica, é considerada como disciplina que estuda as interconexões lógicas entre as proposiçōes descritivas, as quais versam sobre sistemas normativos, bem como as propriedades formais desses sistemas (consistência, inconsistência, trivialidade, ....).

\section{Lógicas Deônticas Não-Clássicas}

Nesta secção exporemos as razões para se construir lógicas deônticas não-clássicas, em especial lógicas deônticas paraconsistentes.

Os códigos morais (e, também, os legais) existentes contêm, normalmente, regras que acarretam contradições: por exemplo, uma ação é obrigatória e, ao mesmo tempo, não é. Ora, se a logica tradicional for utilizada como logica subjacente de um sistema deôntico, por meio do qual sistematiza. mos as proposições desses códigos, somos conduzidos à trivialização, desde que, como é sabido, de proposições contraditóricas a lógica tradicional permite que derivemos um enunciado qualquer. E um fato que a maioria dos sistemas morais e dos legais encerram leis que se contradizem. Então, os códigos morais ou legais em que isso ocorre seriam triviais; seguindo a postura clássica, devemos modificálos, para eliminar suas incongruências.

Porém, há uma outra solução: em vez de se empregar a lógica tradicional como logica subjacente de nosso sistema deôntico, podemos utilizar, em seu lugar, uma lógica paraconsistente. Se assim procedermos, o código que oferecia problemas não precisa ser modificado, e podemos investigá-lo directamente, explicitando sua estrutura formal inconsistente. As contradições nele existentes seriam isoladas, sem se espalhar. Não asseveramos que esta segunda solução seja melhor, pois somente um estudo detalhado da 
ética e do direito pode dar uma resposta a esse problema. Todavia, é interessante que se saiba que há a possibilidade de se estruturar uma lógica deôntica paraconsistente, e que no caso de nos depararmos com contradições em algum código, moral ou legal, com auxílio dessa lógica, não se impõe que o codigo seja modificado.

Outra questão extremamente importante, que nos conduz, também, a uma lógica deôntica paraconsistente, é a questão dos dilemas morais. Uma pessoa enfrenta um dilema moral quando, para ela, é obrigatório efetuar uma ação, mas, ao mesmo tempo, também é obrigatório não efectuá-la. Analogamente, há um dilema moral no caso em que uma ação $\mathbf{A}$, assim como sua não realização, $\sim A$, são proibidas. Estas duas versões dos dilemas morais afiguram-se equivalentes, se certas conexões simples entre os operadores de obrigatoriedade e de proibição forem aceitas.

Um exemplo típico de dilema moral está relacionado com a questão do aborto. Com efeito, suponhamos que um Sr. $X$ esteja no hospital, onde sua esposa vai dar luz, e que o médico lhe informa que ou sua esposa se salva ou o filho. Um dos dois morrerá. $O$ Sr. $X$ pode procurar salvar a mulher, condenando o filho, ou pode tentar salvar o filho, sentenciando sua mulher à morte. Esta situação o defronta com um dilema deôntico, pois ele deve, é obrigatório, salvar uma vida.

São variadas as circunstâncias que nos levam a enfrentar dilemas morais. Não ensaiaremos, aqui, provar que só existem dilemas morais prima facie, ou seja, que uma conveniente ordenação das regras morais evidenciaria que não existem dilemas morais reais. Por outro lado, também, não tentaremos demonstrar que há dilemas verdadeiros. Essas duas tarefas constituem temas de ética, e não de lógica.

No entanto, os dilemas morais estão ligados à lógica. Com efeito, se utilizarmos a lógica deôntica clássica, em uma de suas formulações padrão, mostra-se que a existência de um tal dilema é suficiente para se provar que qualquer proposição é obrigatória. Ou seja, os dilemas causam o colapso deôntico de nosso sistema ético. 0 mesmo se passa se 
empregarmos a maioria dos sistemas clássicos de lógica deôntica.

Então, se admitirmos a existência de dilemas reais, só duas vias estâo abertas para nós: 1) Manter a lógica tradicional, enfraquecendo as leis que governam os operadores deônticos (isto implica no sacrifício de leis básicas, tais como $O(A+B)+(O A+O B))$; 2) Recorremos às lógicas paraconsistentes, para fundamentar os sistemas deônticos, pois elas não excluem, $a b$ initio, os dilemas deônticos reais. A lógica paraconsistente deôntica, que será estudada neste artigo, constitui uma das opções nesta segunda via.

Como os conceitos éticos são um tanto vagos, outra categoria de lógica que se poderia lançar mão no domínio da ética seria uma lógica da vaguidade, talvez paracompleta, simultaneamente.

Há outras motivações para se aplicar lógicas não clássicas no domínio deôntico. Porém, não discutiremos este tópico, em toda sua amplitude, aqui. Limitar-nos-emos, em boa parte, a apresentar alguns sistemas paraconsistentes e deônticos, que permitam que se trate de códigos onde há contradições e nos quais os dilemas morais não se acham excluidos em princípio (sobre estes temas, ver da Costa e Carnielli $198+$, bem como as referências nele contidas). Mas, para tanto, convém antes delinear um sistema clássico, o qual servirá de ponto de partida para a elaboraçao de nosso sistema paraconsistente.

\section{Um Sistema Clássico de Lógica Deôntica}

Nesta secção, introduzimos um sistema clássico de lógica deôntica, baseado no cálculo proposicional clássico $\mathrm{C}_{0}$. Tal sistema, denotado por TD e chamado de sistema $T$ deôntico, é obtido quando acrescentamos à linguagem do cálculo $\mathrm{C}_{0}$ um novo símbolo primitivo 0 , que é um operador monádico chamado de operador de obrigatoriedade, bem como axiomas específicos para o operador 0 . Assim, a linguagem do sistema TD compõe-se dos seguintes símbolos primitivos: 1) conectivos: $\sim$ (negação), $\rightarrow$ (implicação) e 0 
(obrigatório); 2) variáveis: um conjunto infinito enumerável de variáveis proposicionais; e 3) parênteses. Usamos letras maiúsculas latinas para denotar fórmulas e letras maiúsculas gregas para designar conjunto de fórmulas da linguagem do sistema TD. Indicamos por $F$ o conjunto de todas as fórmulas de TD.

Os símbolos ^(conjunção), v(disjunção) e $\longleftrightarrow$ (equivalência) sao definidos do modo usual. Utilizamos, na metamatemática, os símbolos $\Rightarrow$ (implica) e $\Leftrightarrow$ (equivale).

$\mathrm{Na}$ escritura de fórmulas, adotamos convenções óbvias, especialmente no que se refere à supressão de parênteses. Fórmula é definida de modo usual; ou seja: uma variável proposicional é uma fórmula; se A e B são fórmulas, então $\sim A$, OA e $(A \rightarrow B)$ são fórmulas.

Como símbolos definidos temos ainda os seguintes:

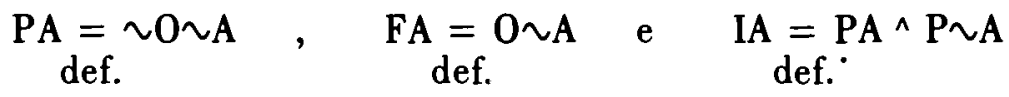

os quais são lidos, respectivamente, 'é permitido que A', 'é proibido que A' e 'é indiferente que A'.

Os postulados de TD (esquemas de axiomas e regras primitivas de inferência) são os seguintes:

$$
\begin{array}{ll}
\mathrm{P}_{1}: A \rightarrow(B \rightarrow A) & \\
\mathrm{P}_{2}:(A \rightarrow B) \rightarrow(A \rightarrow(B \rightarrow C)) \rightarrow(A \rightarrow C) & \\
P_{3}:(\sim B \rightarrow \sim A) \rightarrow((\sim B \rightarrow A) \rightarrow B) \\
P_{4}: O(A \rightarrow B) \rightarrow(O A \rightarrow O B) & \text { (modus ponens) } \\
\mathrm{R}_{1}: \frac{A, A \rightarrow B}{B} \quad \text { (regra de Gödel) } & \\
\mathrm{R}_{2}: \frac{A}{O A} &
\end{array}
$$

Os conceitos de demonstraçao e de teorema são definidos do modo padrão. 
Definição 1 (de dedução com base em $\Gamma$ ): Uma dedução com base em $\Gamma$ é uma sequência finita de fórmulas $A_{1}, A_{2}, \ldots$, $A_{n}$, em que cada $A_{i}$,

$1 \leq \mathrm{i} \leq \mathrm{n}$, é:

1) um axioma; ou

2) pertence a $\Gamma$; ou

3 ) foi obtida de fórmulas precedentes por $R_{1}$ (modus ponens - MP); ou

4) foi obtida de $A_{j}, j<i$, pela aplicação da regra $R_{2}$ e existe uma subsequiência de $A_{1}, A_{2}, \ldots, A_{n}$, que é uma demonstração de $A_{\mathrm{j}}$.

Quando $\Gamma$ é o conjunto vazio, uma dedução com base em $\Gamma$ chama-se demonstração.

Com apoio na Definição 1, pode-se definir o conceito de. consequiência sintática (representado pelo símbolo $\vdash$ ).

Os seguintes teoremas são facilmente provados:

Teorema 1 (da dedução): $\Gamma, \mathrm{A} \vdash \mathrm{B} \Rightarrow \Gamma \vdash \mathrm{A} \rightarrow \mathrm{B}$.

Teorema 2: Valem em TD:

$T_{1}: O A \leftrightarrow \sim P \sim A$

$$
\mathrm{T}_{7}: \mathrm{O} \sim \mathrm{A} \rightarrow \sim \mathrm{OA}
$$

$\mathrm{T}_{2}: \mathrm{O}(\mathrm{A} \wedge \mathrm{B}) \leftrightarrow(\mathrm{OA} \wedge \mathrm{OB}) \quad \mathrm{T}_{8}: \mathrm{P}(\mathrm{A} \rightarrow \mathrm{B}) \leftrightarrow(\mathrm{OA} \rightarrow \mathrm{PB})$

$\mathrm{T}_{3}: \mathrm{P}(\mathrm{A} \vee \mathrm{B}) \leftrightarrow(\mathrm{PA} \vee \mathrm{PB}) \quad \mathrm{T}_{9}: \mathrm{P}(\mathrm{A} \wedge \sim \mathrm{A}) \rightarrow(\mathrm{A} \wedge \sim \mathrm{A})$

$\mathrm{T}_{4}: \mathrm{O}(\mathrm{A} \vee \mathrm{B}) \rightarrow(\mathrm{PA} \vee \mathrm{OB}) \quad \mathrm{T}_{10}:(\mathrm{PA} \wedge \mathrm{OB}) \rightarrow \mathrm{P}\left(\mathrm{A}^{\wedge} \mathrm{B}\right)$

$\mathrm{T}_{5}:(\mathrm{OA} \vee \mathrm{OB}) \rightarrow \mathrm{O}(\mathrm{A} \vee \mathrm{B}) \quad \mathrm{T}_{11}: O A \wedge \mathrm{OAA} \rightarrow \mathrm{B}$

$\mathrm{T}_{6}: \mathrm{P}(\mathrm{A} \wedge \mathrm{B}) \rightarrow(\mathrm{PA} \wedge \mathrm{PB}) \quad \mathrm{T}_{12}: \mathrm{FA} \wedge \mathrm{F} \sim \mathrm{A} \rightarrow \mathrm{B}$

Teorema 3: Sendo $O \Gamma=\{O B: B \in \Gamma\}$, então:

$$
\Gamma \vdash \mathrm{A} \Rightarrow \mathrm{O} \Gamma \vdash \mathrm{OA}
$$


Definição 2: Um conjunto $\Gamma$ é inconsistente em TD se existe uma fórmula $\mathrm{A} \in F$ tal que $\Gamma \vdash \mathrm{A}$ e $\Gamma \vdash \sim A$; em caso contrário, $\Gamma$ é consistente.

Definição 3: (de $\mathrm{C}_{0}$-transformada): Seja $\mathrm{A} \in F$. Dizemos que $\mathrm{A}^{*}$ é a $\mathrm{C}_{0}$-transformada de $\mathrm{A}$ se $\mathrm{A}^{*}$ pode ser obtida de $\mathrm{A}$ suprimindo-se todas as ocorrências do símbolo 0 .

Teorema 4: Sejam $\mathrm{A} \in F$ e $\mathrm{A}^{*}$ sua $\mathrm{C}_{0}$-transformada. Então: $\vdash \mathrm{A}$ em TD $\Rightarrow \vdash \mathrm{A}^{*}$ em $\mathrm{C}_{0}$.

Teorema 5: 0 sistema TD é consistente.

Demonstração: Conseqüência do Teorema 4 e da consistência do cálculo $\mathrm{C}_{0}$.

A seguir, formularemos uma semântica para TD e mostraremos que, relativamente a essa semântica, TD é correto e completo.

Definição 4: (de TD-estrutura): Uma estrutura deôntica para TD ou, simplesmente, uma TD-estrutura, é uma tripla $<W$, $\mathbf{R}, \Vdash \vdash$, onde $W$ é um conjunto não vazio de elementos, $R$ é um subconjunto de $W X W$, isto é, uma relação binária entre os elementos de $W$, tal que, para todo $w \in W$, existe $w^{\prime} \in \mathbb{W}$ satisfazendo a condição de que $w R w^{\prime}$, e $\Vdash$ é uma relação entre os elementos de $\mathbb{W}$ e as fórmulas de TD de modo que se tenha:

1) $w \Vdash t A \quad \Leftrightarrow \quad \Vdash \forall \sim A$ (negação de $w$ i $A$ )

2) $w \Vdash A \rightarrow B \Leftrightarrow w \quad H A$ ou $w \Vdash B$

3) $w \Vdash O A \quad \Leftrightarrow w^{\prime} \Vdash A$, para todo $w^{\prime} \in W$ tal que $w$ $\mathrm{R}$ w'.

Os elementos de W são chamados de mundos. Quando os mundos w e w' de W são relacionados por $R$, o que indica- 
mos por wRw', esta expressão é lida 'w' é acessível deonticamente a w'. A relação $\mathrm{R}$ é chamada de relação de acessibilidade entre os mundos de W. A relação It denomina-se relação de $T D$-forçamento. As expressões w $\Vdash A$ e w $\Vdash$ A são lidas, respectivamente, 'w força $A$ ' e 'w não força $A$ '.

Definição 5: sejam $<\mathrm{W}, \mathrm{R}, \Vdash>>$ uma TD-estrutura, w $\in$ $\mathrm{W}, \mathrm{A} \in F$ e $\Gamma \in F$. Então:

1) A é válida em um mundo w se w $\Vdash A$;

2) A é válida em uma TD-estrutura se $A$ é válida em w, para todo $w \in \mathbb{W}$;

3) A é válida no sistema TD, ou, simplesmente, A é TD. válida, se A é válida em toda TD-estrutura;

4) w é um modelo de $\Gamma$ se,para toda $B \in \Gamma$, B é válida em w.

Com base na Definição 5, pode-se definir o conceito de consequiência semântica $(\vDash)$.

Facilmente se demonstra o seguinte teorema:

Teorema 6 (da correção): Seja $\Gamma \mathrm{U}\{\mathrm{A}\} \in F$; então, no sistema TD, tem-se:

$$
\Gamma \vdash \mathbf{A} \Rightarrow \Gamma=\mathbf{A}
$$

Definição 6 (de conjunto maximal consistente): $\Gamma$ é maximal consistente em TD se $\Gamma$ é consistente e, para toda fórmula $A \notin \Gamma, \Gamma \mathrm{U}\{\mathrm{A}\}$ é inconsistente em TD.

Definição 7: Sejam A, B $\in F$ e façamos

$M=\{\Delta: \Delta$ é maximal consistente $\}$, def.

$\Gamma^{0}=\{A \in \Gamma:$ para alguma $B, A=O B\}$, def. 
$\epsilon\left(\Delta^{0}\right)=\{A: O A \in \Delta\}$, def.

$S=$ o subconjunto de MXM, tal que: $\Delta S \Delta^{\prime} \Leftrightarrow \epsilon\left(\Delta^{0}\right) \subset \Delta^{\prime}$. def.

Lema 1: $\Gamma$ é consistente $\Rightarrow \epsilon\left(\Gamma^{0}\right)$ é consistente.

Demonstração: Suponhamos que $\epsilon\left(\Gamma^{0}\right)$ seja inconsistente e que A seja uma fórmula qualquer do sistema TD; então:

1) $\epsilon\left(\Gamma^{0}\right) \vdash A^{\wedge} \sim A$

2) $\Gamma^{0} \vdash O\left(A^{\wedge} \sim A\right)$

3) $\Gamma \vdash O\left(A^{\wedge} \sim A\right)$

4) $\Gamma \vdash O A^{\wedge} O \sim A$

5) $\Gamma \vdash O A$

6) $\Gamma \vdash O \sim A$

7) $\Gamma \vdash O \sim A \rightarrow \sim O A$

8) $\Gamma \vdash \sim 0 A$

9) $\Gamma$ é inconsistente

Lema 2 (de Lindenbaum): Todo conjunto consistente de fórmulas de TD está contido num conjunto maximal consistente.

Demonstração: E feita de modo análogo ao usual.

Lema 3: Sejam A, B $\in F$. Se $\Delta$ é maximal consistente, entao:

1) $A \in \Delta \Leftrightarrow \Delta \vdash A$

2) $\sim A \in \Delta \Leftrightarrow A \notin \Delta$

3) $\mathrm{A} \rightarrow \mathrm{B} \in \Delta \Leftrightarrow \mathrm{A} \notin \Delta$ ou $\mathrm{B} \in \mathrm{A}$

4) $\mathrm{OA} \in \Delta \Rightarrow \mathrm{O} \sim \mathrm{A} \notin \Delta$

Lema 4: Para todo $\Delta \in \mathbf{M}$, existe $\Delta^{\prime} \in \mathbf{M}$, de modo que $\Delta \mathrm{S} \Delta^{\prime}$. 
Demonstração: Admitamos que $\Delta \in \mathbf{M}$; portanto, $\Delta$ é consistente e, conseqüentemente, pelo Lema $1, \epsilon\left(\Delta^{0}\right)$ é consistente. Assim, pelo Lema 2, existe $\Delta^{\prime}$ maximal consistente, tal que $\epsilon\left(\Delta^{0}\right) \subset \Delta^{\prime}$; donde $\Delta S \Delta^{\prime}$, pela Definição 7 .

Nosso objetivo, a seguir, é construir uma TD-estrutura. Para isso, a cada $\Delta \in \mathbf{M}$ associaremos um mundo, o qual in. dicaremos por $w_{\Delta}$. Seja $\bar{W}$ o conjunto formado por todos esses mundos. Seja $\hat{\mathbf{R}}$ uma relação entre os mundos de $\overline{\mathrm{W}}$ tal que $w_{\Delta} \tilde{\mathbf{R}} w_{\Delta}$, se, e somente se, $\Delta \mathbf{S} \Delta^{\prime}$. Consideremos a relação $\Vdash$ dada por $w_{\Delta} \Vdash A$ se, e somente se, $A \in \Delta$.

Facilmente, se pode verificar que $\overline{\mathrm{W}}$ é un conjunto não vazio; que a relação $\dot{R}$ satisfaz a propriedade de que para todo $w \in \bar{W}$, existe $w^{\prime} \in \bar{W}$ tal que $w \bar{R}$ w' (pelo Lema 4); e, finalmente, que a relação $\Vdash$ é uma relação de TDforçamento (Lema 3).

Assim, a tripla $<\overline{\mathrm{W}}, \overline{\mathrm{R}}, \Vdash>$ é uma TD-estructura para o sistema TD, chamada TD-estrutura canônica.

Teorema 7: A todo conjunto consistente de fórmulas de TD está associada uma TD-estru tura, sendo todas as suas fórmulas válidas num mundo dessa TD-estrutura.

Demonstração: Basta considerar a TD-estrutura canônica.

Teorema 8 (da completude): Seja $\Gamma \mathrm{U}\{\mathrm{A}\} \subset F$. Então, no sistema TD, tem-se:

$$
\Gamma \models A \Rightarrow \Gamma \vdash A
$$

Demonstração: Consequiência do Teorema 7.

Observe-se que um dilema moral trivializa TD, o que decorre do Teorema 2 , por $\mathrm{T}_{11}$ ou $\mathrm{T}_{12}$. Além disso, é óbvio que esse sistema não pode ser o fundamento lógico de códigos inconsistentes. 


\section{Lógica Deôntica Paraconsistente}

$\mathrm{Na}$ secção 4, se ao invés de tomarmos o cálculo proposicional clássico $\mathrm{C}_{0}$ como lógica subjacente de nossa lógica deôntica, tomarmos o cálculo proposicional paraconsistente $\mathrm{C}_{1}$ (da Costa 1974), obteremos um sistema de lógica deôntica paraconsistente, o qual denotamos por $\mathrm{TD}_{1}$. A linguagem do sistema $\mathrm{TD}_{1}$ compõe-se dos seguintes símbolos primitivos: 1) conectivos: (negação), *(implicação), v(disjunçaō), ^(conjunção) e $\mathrm{O}$ (obrigatório); 2) variáveis proposicionais; $\mathrm{e}$ 3)parênteses. $O$ símbolo $\rightarrow$ (equivalência) é definido como na lógica clássica, assim como os conceitos de fórmula, de demonstração, de teorema e de dedução com base em $\Gamma$ (cf. secção 4, Definição 1).

Os postulados de $\mathrm{TD}_{1}$ são os seguintes:

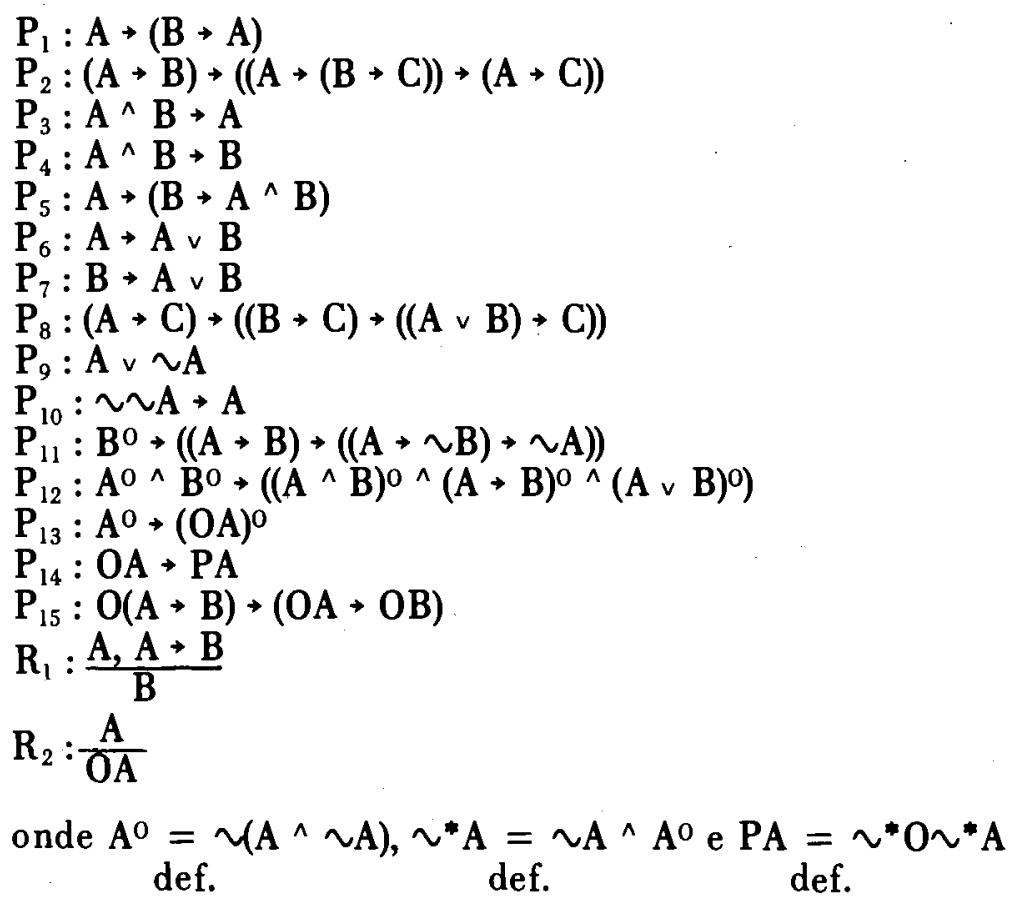


Os seguintes teoremas são facilmente provados:

Teorema 9 (da dedução): $\Gamma, \mathrm{A} \vdash \mathrm{B} \Rightarrow \Gamma \vdash \mathrm{A} \rightarrow \mathrm{B}$.

Teorema 10: Valem em $\mathrm{TD}_{1}$ :
$\mathrm{OA} \rightarrow \sim * \mathrm{P} \sim * A$
$A^{0} \rightarrow \sim(O A \wedge O \sim A)$
$(O A \wedge O \sim * A)+O B$
$\left(O \sim A^{\wedge} A^{0}\right) \rightarrow \sim O A$
$\sim^{*}\left(O A^{\wedge} \sim * O A\right)$
$(\mathrm{OA}+\mathrm{OB})+(\sim \mathrm{OA} \vee \mathrm{OB})$

Teorema 11: 0 s seguintes esquemas nào valem em $\mathrm{TD}_{1}$ :

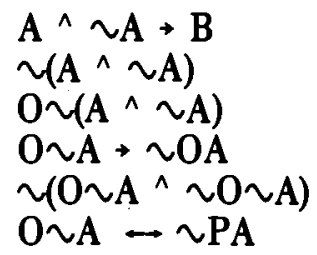

$A \rightarrow \sim \sim A$

$\sim A \wedge \sim B \rightarrow \sim(A \vee B)$

$\sim A \vee \sim B \rightarrow \sim(A \wedge B)$

$(A \rightarrow B) \rightarrow(\sim B \rightarrow \sim A)$

$(O A \wedge O \sim A) \rightarrow B$

$(O A \wedge O \sim A) \rightarrow O B$

Definição 8: Um conjunto $\Gamma$ é trivial em $\mathrm{TD}_{1}$ se para toda fórmula $A, \Gamma \vdash A$; em caso contrário, $\Gamma$ é não-trivial em $T_{1}$.

Teorema 12: $\Gamma$ é trivial $\Rightarrow \Gamma$ é inconsistente.

A recíproca deste teorema não vale de modo geral num sistema paraconsis tente; contudo, nos sistemas clássicos trivialidade e inconsistência săo noções equivalentes.

De modo análogo ao da secção 4 , pode-se definir o conceito de $\mathrm{C}_{1}$-transformada; prova-se que se uma fórmula $A$ é um teorema em $\mathrm{TD}_{1}$, então a sua $\mathrm{C}_{1}$-transformada $A^{*}$ é, também, um teorema $\mathrm{C}_{1}$. Demonstra-se, também, que o sistema $\mathrm{TD}_{1}$ é consistente e, além disso, que $\mathrm{TD}_{1}$ é extensão conservativa de $\mathrm{C}_{1}$.

Definiçao 9 (de 0-deonticamente trivializável): Uma fórmula $A$ de $\mathrm{TD}_{1}$ trivializa 0 -deonticamente o sistema $\mathrm{TD}_{1}$ se, para toda fórmula $\mathrm{B}, \vdash \mathrm{A} \rightarrow \mathrm{OB}$ em $\mathrm{TD}_{1}$. 
Teorema 13: 0 sistema $\mathrm{TD}_{1}$ não é 0 -deonticamente trivializável por uma fórmula do tipo $\mathrm{OA}{ }^{\wedge} \mathrm{O} \sim \mathrm{A}$.

Demonstração: Suponhamos que $\mathrm{TD}_{1}$ seja 0 -deonticamente trivializável por uma fórmula do tipo $O A^{\wedge} 0 \sim A$. Isso significa que, em $\mathrm{TD}_{1}, \vdash\left(O \mathrm{~A}^{\wedge} \mathrm{O} \sim \mathrm{A}\right) \rightarrow \mathrm{OB}$, para toda fórmula $\mathrm{B}$; conseqüentemente, $\vdash\left(A^{\wedge} \sim A\right) \rightarrow B$ em $C_{1}$ e, portanto, pelo Teorema da extensao conservativa, $\vdash\left(A^{\wedge} \sim A\right) \rightarrow B$ em $T_{1}$, o que não é possível pelo Teorema 11.

A seguir, descrevemos uma semântica para $\mathrm{TD}_{1}$. Consideremos $\langle\mathbb{W}, R, \Vdash\rangle$, onde $W$ e $R$ são definidos como na seç̧ão 4 e $\Vdash$ é uma relação de forçamento (entre mundos de $W$ e fórmulas de $T D_{1}$ ), satisfazendo as seguintes condições:

1) $\mathrm{w} \Vdash \mathrm{A} \Rightarrow \mathrm{w} \Vdash \sim \mathrm{A}$

2) $w \Vdash \sim \sim A \Rightarrow w$ $\Vdash A$

3) $w \Vdash A \rightarrow B \Leftrightarrow w \Vdash A$ ou $w \Vdash B$

4) $w \Vdash A \vee B \Leftrightarrow w \Vdash A$ ou $w \Vdash B$

5) $w \Vdash A \wedge B \Leftrightarrow w \Vdash A$ e $w \Vdash B$

6) $w \Vdash A^{0} \wedge B^{0} \Rightarrow w \Vdash(A \rightarrow B)^{0} \wedge(A \wedge B)^{0} \wedge(A \vee B)^{0}$

7) $w \Vdash B^{0}, w \Vdash A \rightarrow B$ e w $\Vdash A \rightarrow \sim B \Rightarrow w_{\|}$

8) $w \Vdash t A^{0} \Rightarrow w \vdash(O A)^{0}$

9) $w \Vdash O A \Leftrightarrow w$, $\Vdash A$, para todo w' $\in \mathbb{W}$, tal que $w R$ w'

A tripla $<\mathrm{W}, \mathrm{R}, \Vdash>>$ é chamada uma $T D_{1}$-estrutura.

Lema 5: Seja $<\mathrm{W}, \mathrm{R}, \Vdash>>$ uma $\mathrm{TD}_{1}$-estrutura. Para todo w $\in W$ e toda formula $A$, tem-se:

$$
\mathrm{w} \Vdash A \Leftrightarrow w \Vdash \sim * A
$$

Demonstração: Suponhamos que w $\Vdash$ A e w $\Vdash \sim * A$. Se w

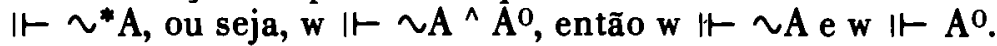
Mas, como w $\Vdash A$, decorre que $w \Vdash A \rightarrow A, w \Vdash A \rightarrow \sim A$ e w $\Vdash A^{0}$; conseqüentemente, pela Definição de $T_{1^{-}}$. estrutura (item 7), w $\Vdash$ A o que não é possível. Reciproca- 
mente, se $w \| H \sim * A$, isto é w $\| \nvdash \sim A^{\wedge} A^{0}$, então $w \| H \sim A$ ou $w$ It $A^{0}$. Temos dois casos para analisar: 1) Se $w$ It $\sim A$, advém que $w \Vdash \sim \sim A$ e, portanto, $w \Vdash A .2)$ Se $w \Vdash A^{0}$, isto é, $w \Vdash \mid \neg \sim\left(A^{\wedge} \sim A\right)$, decorre que $w \Vdash A^{\wedge} \sim A$ e, por conseguinte, $w \Vdash A$.

Lema 6 (de Lindenbaum): Todo conjunto de fórmulas de $\mathrm{TD}_{1}$ não-trivial está contido num conjunto não-trivial maximal.

A seguir usamos as mesmas notações introduzidas na secção 4, com adaptações óbvias.

Lema 7: $\Gamma$ é não-trivial $\Rightarrow \epsilon\left(\Gamma^{0}\right)$ é não-trivial.

Demonstração: Suponhamos que $\epsilon\left(\Gamma^{0}\right)$ é trivial e sejam A e $\mathrm{B}$ fórmulas quaisquer do sistema $\mathrm{TD}_{1}$; então:

1) $\epsilon\left(\Gamma^{0}\right) \vdash A^{\wedge} \sim^{*} A$

2) $\Gamma^{0} \vdash O\left(A^{\wedge} \sim^{*} A\right)$

3) $\Gamma \vdash O A^{\wedge} O O^{*} A$

4) $\Gamma \vdash O A$

5) $\Gamma \vdash O \sim * A$

6) $\quad \vdash \mathrm{O} \sim \mathrm{A}+\sim * \mathrm{OA}$

7) $\Gamma \vdash \sim * O A$

8) $\Gamma \vdash \mathrm{OA}^{\wedge} \sim * \mathrm{OA}$

9) $\vdash\left(\mathrm{OA}^{\wedge} \sim^{*} \mathrm{OA}\right) \rightarrow \mathrm{B}$

10) $\Gamma \vdash \mathrm{B}$

11) $\Gamma$ é trivial

Lema 8: Se $\Delta$ é não-trivial maximal, tem-se:

1) $A \in \Delta \Leftrightarrow \Delta \vdash A$

2) $A \rightarrow B \in \Delta \Leftrightarrow A \notin \Delta$ ou $B \in \Delta$

3) $A^{\wedge} B \in \Delta \Leftrightarrow A \in \Delta$ e $B \in \Delta$

4) $A \vee B \in \Delta \Leftrightarrow A \in \Delta$ ou $B \in \Delta$

5) $A \in \Delta \Leftrightarrow \sim * A \notin \Delta$

6) $A \in \Delta$ e $A^{0} \in \Delta \Rightarrow \sim A \notin \Delta$

7) $\sim \mathrm{A} \in \Delta$ e $\mathrm{A}^{0} \in \Delta \Rightarrow \mathrm{A} \notin \Delta$

8) $\mathrm{A}^{\wedge^{\wedge}} \mathrm{B}^{0} \in \Delta \Rightarrow(\mathrm{A} \rightarrow \mathrm{B})^{\circ} \in \Delta,(\mathrm{A} \vee \mathrm{B})^{0} \in \Delta \mathrm{e}\left(\mathrm{A}^{\wedge} \mathrm{B}\right)^{\circ} \in \Delta$ 
9) $A^{0} \in \Delta \Rightarrow(\sim A)^{0} \in \Delta$

10) $O A \in \Delta \Rightarrow O \sim A \notin \Delta$

11) $A^{0} \in \Delta \Rightarrow(O A)^{0} \in \Delta$

12) $\mathrm{B}^{\circ} \in \Delta, \mathrm{A} \rightarrow \mathrm{B} \in \Delta \mathrm{e} \mathrm{A} \rightarrow \sim \mathrm{B} \in \Delta \Rightarrow \mathrm{A} \notin \Delta$.

Lema 9: Para todo $\Delta \in \mathbf{M}$, existe $\Delta^{\prime} \in \mathrm{M}$, de maneira que $\Delta \mathrm{S} \Delta^{\prime}$.

Teorema 14 (da correção e completude): Seja $\Gamma$ U\{A\} um conjunto de fórmulas de $\mathrm{TD}_{1}$. Então, $\Gamma \vdash \mathrm{A} \Leftrightarrow \Gamma \models A$.

Demonstração: Conseqüência dos lemas precedentes.

Pelo Teorema 13 , verifica-se que $\mathrm{TD}_{1}$ não exclui, em princípio, os dilemas morais. Por outro lado, ele pode servir de fundamento para códigos morais que encerrem contradições, como é patente.

\section{Observações Finais}

$\mathrm{TD}_{1}$ é um sistema paraconsistente de lógica deôntica. Como vimos, ele não elimina os dilemas deônticos por motivos 'lógicos'. Ao contrário, tais dilemas podem ser manipulados por seu intermédio, sem perigo de trivialização. Mais ainda, por ser paraconsistente, $\mathrm{TD}_{1}$ pode servir de base para codigos, morais ou legais, sem que se necessite modificá-los, para se contornar as contradições porventura existentes.

Assim, $\mathrm{TD}_{1}$ constitui-se numa alternativa para sistemas clássicos, como TD, os quais, em certo sentido, vetam a inconsistência e a possibilidade de dilemas reais.

Ficam abertas, pois, como já observamos, duas vias para quem se ocupa da ética e do direito: adotar a logica tradicional, e eliminar, $a b$ initio, as contradições e os dilemas, ou empregar uma lógica, como a paraconsistente, para não deixar de lado, de saída, contradições e dilemas. A escolha da melhor dentre estas duas vias só poderá ser feita mediante investigações éticas e de teoria do direito; não é questão 
puramente lógica. 0 lógico, qua lógico, somente pode apontar caminhos alternativos do prisma lógico.

Convém notar que tanto $\mathrm{TD}$ como $\mathrm{TD}_{1}$ são susceptíveis de ser estendidos a cálculos de primeira ordem e a lógicas de ordem superior (ver, por exemplo, Puga 1985).

\section{REFERENCIAS}

Arruda, A.I. (1980) 'A survey of paraconsistent logic', in Mathematical Logic in Latin América, A. I. Arruda, R. Chuaqui and N.C.A. da Costa, eds., North-Holland, pp. 1-41.

Da Costa, N.C.A. (1974) 'On the theory of inconsistent formal systems', Notre Dame Journal of Formal Logic 15, 497-510.

Da Costa, N.C.A. e W.A. Carnielli $(198+)^{\prime}$ 'On Paraconsistent Deontic Logic', a aparecer em Philosophia.

Hilpinen, R. (1971) Deontic Logic: Introductory and Systematic Readings, Reidel.

Hilpinen, R. (1981) New Studies in Deontic Logic, Reidel.

Loparic, A. e N.C.A. da Costa (1984) 'Paraconsistency, paracompleteness and valuations', Logique et Analyse 106, 119-131.

Puga, L.Z. (1985) Uma Logica do Querer, Tese de Doutorado, Pontifícia Universidade Católica de São Paulo.

Puga, L.Z., N.C.A. da Costa e W.A. Carnielli $(198+)^{~ ' K a n t i a n ~ a n d ~ n o n-~}$ Kantian Logics', a aparecer. 
Our starting point, in this basically expository paper, is the study of a classical system of deontic propositional logic, classical in the sense that it constitutes an extension of the classical propositional calculus. It is noted, then, that the system excludes ab initio the possibility of the existence of real moral dilemmas (contradictory obligations and prohibitions), and also can not cope smoothly with the so-called prima facie moral dilemmas. So, we develop a non-classical, paraconsistent system of propositional deontic logic which is compatible with such dilemmas, real or prima facie. In our paraconsistent system one can handle them neatly, in particular one can directly investigate their force, operational meaning, and the most important consequences of their acceptance as not uncommon moral facts.

Of course, we are conscious that other procedures for dealing with them are at hand, for example by the weakening of the specific deontic axioms. It is not argued that our procedure is the best, at least as regards the presert state of the issue. We think only that owing, among other reasons, to the circumstance that the basic ethical concepts are intrinsically vague, it seems quite difficult to get rid of moral dilemmas and of moral deadlocks in general. Apparently this speaks in favour of a paraconsistent approach to ethics. At any rate, a final appraisal of the possible solutions to the problem of dilemmas and deadlocks, if there is one, constitutes a matter of ethical theory and not only of logic. On the other hand, the paraconsistency stance looks likely to be relevant also in the field of legal logic.

It is shown, in outline, that the systems considered are sound and complete, relative to a natural semantics. All results of this paper can be extended to first-order and to higher-order logics. Such extensions give rise to the question of the transparency (or oppacity) of the deontic contexts. As we shall argue in forthcoming articles, they normally are transparent. 